\title{
Improvement of Robotic Micromanipulations Using Chemical Functionalisations
}

\author{
Jérôme Dejeu, Patrick Rougeot, Michaël Gauthier, and Wilfrid Boireau \\ FEMTO-ST Institute, UMR CNRS 6174 - UFC/ENSMM/UTBM, \\ 24 rue Alain Savary, 25000 Besançon, France \\ Firstname.surnamed femto-st.fr
}

\begin{abstract}
Robotic microhandling is disturbed by the adhesion phenomenon between the micro-object and the grippers. This phenomenon is directly linked to both the object and the gripper surface chemical composition. We propose to control adhesion by using chemical self-assembly monolayer (SAM) on both surfaces. Previous distance-force measurements done with AFM have shown that the liquid $\mathrm{pH}$ can be used to modify the adhesion and created repulsive force between the gripper fingers and the micro-objet. This paper shows the correlation between the force distance distance measurements and the micromanipulation tasks using chemically functionalized grippers.
\end{abstract}

Keywords: Microhandling, Surface functionalisation, Aminosilane, Adhesion, Pull-off forces.

\section{Introduction}

The assembly of microsystems is a great challenge because of the microscopic size of the components. In fact, the major difficulty of micro-assembly comes from the micro-objects' behaviour which depends on surface forces [1-2]. The manipulation of a micro-object requires handling, positioning, and releasing without disturbances of the surface forces such as electrostatic forces, van der Waals forces or capillary forces. The release is the most critical phase which is usually hindered by adhesion.

Several methods have been proposed in the last ten years to improve micromanipulation. The first approach consists in using non-contact manipulation like laser trapping or dielectrophoresis. These manipulation methods are not disturbed by adhesion but the blocking force stays low and thus cannot be easily applied microassembly. The second approach deals with contact manipulation, where the adhesion is directly used for manipulation or strategies, is used to reduce adhesion between end-effectors and objects.

In this article, we propose a new contact handling system based on a chemical control of the surface forces between the object and the gripper. The objective is to control the adhesion force or to create a repulsive force to guarantee a reliable release. Now, the surface properties of a material can be controlled by surface functionalisation in a liquid by modifying the $\mathrm{pH}$. The charge density on functionalized surfaces is effectively linked to the $\mathrm{pH}$. The microhandling principle based on chemical switching surface is presented in figure 1 . The grasping can be done at $\mathrm{pH} 1$ where the surface charge on the 
gripper and the object induces an attractive force. In order to release the object, the $\mathrm{pH}$ is modified to a second value $\mathrm{pH} 2$ where the object charge is changing. The electrostatic force becomes repulsive and the object is released.

This article deals with comparative handling operations performed with different chemical functionalisations at different $\mathrm{pH}$.
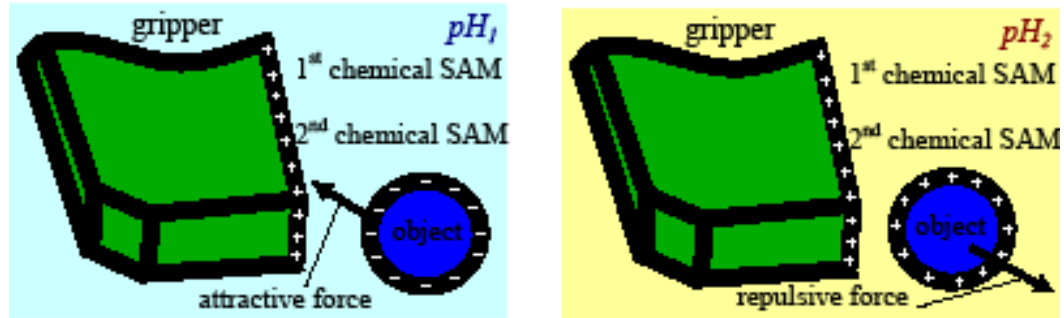

Fig. 1. Principle of the Robotic Microhandling controlled by Chemical Self Assembly Monolayer (SAM)

\section{Chemical Functionalisation}

\subsection{General Principles}

The microhandling method proposed is based on two chemical functions: amine and silica. In one hand, the amino group is in state $\mathrm{NH}_{2}$ in basic $\mathrm{pH}$ and in $\mathrm{NH}_{3}{ }^{+}$in acidic $\mathrm{pH}$. In the other hand, the silica surface charge in water is naturally negative, excepted for very acidic $\mathrm{pH}$, where the surface is weakly positive [3].

The surface functionalisation of both object and gripper can be obtained by different methods. The two most important methods are the molecules grafting on the surface (covalent bond between the substrate and the molecules) or the polyelectrolyte physisorption (polyelectrolyte with positive or negative charges) [4].

We chose to investigate the first method because, firstly, it generates covalent bond between substrate and molecules. These molecules must contain silanol, thiol, azide, allyl or vinyl groups in one extremity. These molecules have to be used in organic solvent such as toluene, acetone, methanol, ethanol, etc. The silanol creates a $\mathrm{Si}-\mathrm{O}-\mathrm{Si}$ bond with the silica substrate while allyl or vinyl generates $\mathrm{Si}-\mathrm{O}-\mathrm{C}$ (or $\mathrm{Si}-\mathrm{C}$ ) bond and the acide groups produce $\mathrm{Si}-\mathrm{N}$ bond [5]. The second reason is that the layer created by silanisation did not exhibit any signature of degradation when stored in an airtight container for 18 months [6], and was stable up to a temperature near $350^{\circ} \mathrm{C}$ [7] and when washed using $1 \%$ detergent solution, hot tap water or organic solvents and aqueous acid at room temperature [7].

\subsection{Chemical Components}

The chemical compounds (APTES) used to surface functionalisation are amine functions $\mathrm{NH}_{2}$ which can protonated or ionised to $\mathrm{NH}_{3}{ }^{+}$according to $\mathrm{pH}$. In acidic $\mathrm{pH}$, the amine is totally ionised, then the ionisation decreases and is null in basic $\mathrm{pH}$ (between $\mathrm{pH} 9$ and 12). 
The silanes (APTES), ethanol, sodium chloride $(\mathrm{NaCl})$, sodium hydroxyde $(\mathrm{NaOH})$ and chlorydric acid $(\mathrm{HCl})$, were purchased from Sigma Aldrich.

\subsection{Functionalisation Mechanisms: Grafted Silanes}

The self assembled monolayers formation mechanism during silanization process takes place in four steps [6]. The first step is physisorption, in which the silane molecules get physisorbed at the hydrated silicon surface. In the second step, the silane head-groups comes close to the substrate hydrolyse, in the presence of the adsorbed water layer on the surface, into highly polar hydroxysilane $-\mathrm{Si}(\mathrm{OH})$. These polar $\mathrm{Si}(\mathrm{OH})$ groups form covalent bonds with the hydroxyl groups on $\mathrm{SiO}_{2}$ surface (third step). At least, condensation reaction (release of water molecules) appears between silanol functions and neighbor molecules. Self assembly is driven by lipophilic interactions between the linear alkane moieties.

During initial period, only a few molecules will adsorb (by steps 1-3) on the surface and the monolayer will definitely be in a disordered (or liquid) state. However, at long term, the surface coverage eventually reaches the point where a well-ordered and compact (or crystalline) monolayer is obtained (step 4).

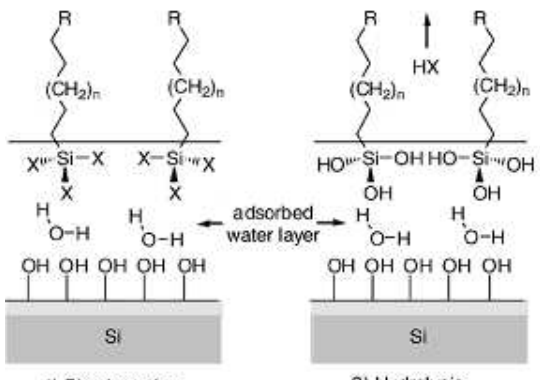

1) Physisorption $(\mathrm{X}=\mathrm{Cl}, \mathrm{OEt}, \mathrm{OMe})$

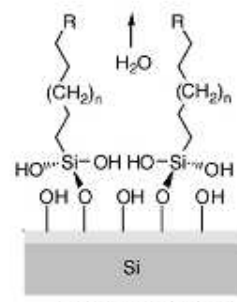

3) Covalent grafting to the substrate

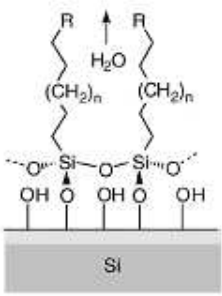

4) In plane reticulation

Fig. 2. Steps involved in the mechanism of SAM formation on a hydrated silicon surface [6]

\subsection{Characterisation of the Fonctionalised Surfaces}

Force measurements were performed in order to characterize the functionalisations [8-9]. Force-distance curves were performed using a stand-alone SMENA scanning probe microscope (NT-MDT).

The force measurement performed on this Atomic Force Microscope (AFM) is based on the measurement of the deformation of the AFM cantilever with a laser deflection sensor. The silicon rectangular AFM cantilever, whose stiffness is $0.3 \mathrm{~N} / \mathrm{m}$, was fixed and the substrate moved vertically. As the applicative objective of this work is to improve reliability of micro-object manipulation, interactions have been studied between a micrometric sphere and a plane. Measurements were in fact performed with a cantilever where a borosilicate sphere $\left(r_{2}=5 \mu \mathrm{m}\right.$ radius) was glued (company Novascan Technologies, Ames, USA).

All measurements were done at the driving speed of $200 \mathrm{~nm} / \mathrm{s}$ to avoid the influence of the hydrodynamic drag forces [10]. For each sample, nine measures were 
done in different points. The repeatability of all the pull-off and pull-in forces values was better than $10 \%$.

\section{Micromanipulation System}

The impact of the functionalisation has been tested on a robotic micro-assembly device composed of a robotic structure, optical microscope, a piezogripper.

Our current robotic micro-assembly device is presented in figure $\operatorname{ref}\{$ Fig:Station $\}$. Actuation is divided into two groups which have 3 degrees of freedom (DOF). The first one allows displacement of the substrate, where microparts are placed. Two linear and one rotation DOFs are available in the horizontal plane. The second group is a 'robotic arm', composed of three linear DOFs and two rotation DOFs which enable displacements and rotations of the microgripper.

Moreover, the microgripper is based on a 4 DOFs piezoelectric actuator with silicon finger tips [11]. This gripper induces motions with a great resolution (few tenth of nanometers).

Experiments have been done in wet medium with a functionalised object and :

- $\quad$ end-effectors grafted with APTES and APDMES or

- non-functionalised end-effectors.

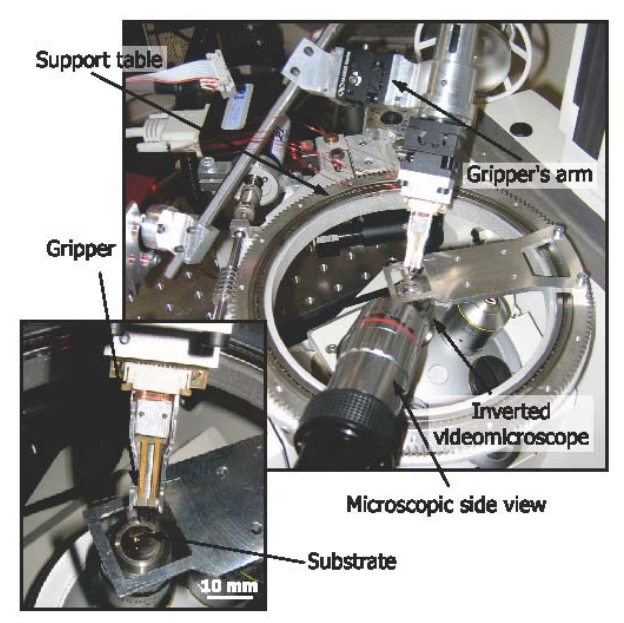

Fig. 3. Microassembly robotic device

Microscopical vision is provided by two videomicroscopes. As the volume above the micromanipulation plane is dedicated to microgripper movement, an inverted microscope LEICA DM-IRBE is used. It enables micro-assembly in liquid medium, whose general interest is synthesized in [2]. A second view for teleoperated operations is given by a side videomicroscope. 


\section{Micromanipulation Results}

\subsection{Non-functionalized Objects}

The micromanipulation tasks are often disturbed by the adhesion force between the grippers and the micro-object. Indeed, with the AFM, we measure a pull-off greater than $1 \mu \mathrm{N}$ (Figure 4). This important adhesion force is confirmed by the micromanipulation task with the micromanipulation system. The glass sphere between 1 and 45 $\mu \mathrm{m}$ stay attach to the end-effector of the gripper when we open the gripper (Figure 5). The glass spheres are as glued on the end-effector whoever the glass size. Indeed, in the range from 1 to $45 \mu \mathrm{m}$, we observe the same phenomena, no release of the glass when we open the gripper.

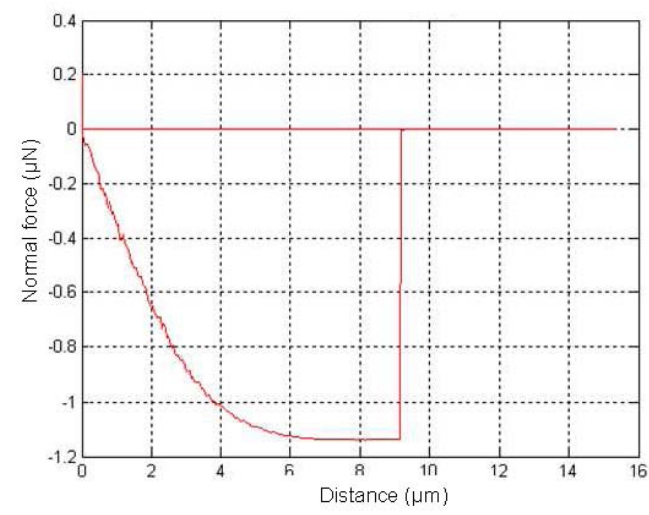

Fig. 4. Force-distance curve between borosilicate cantilever and silica surface (spring constant $0.3 \mathrm{~N} / \mathrm{m})$
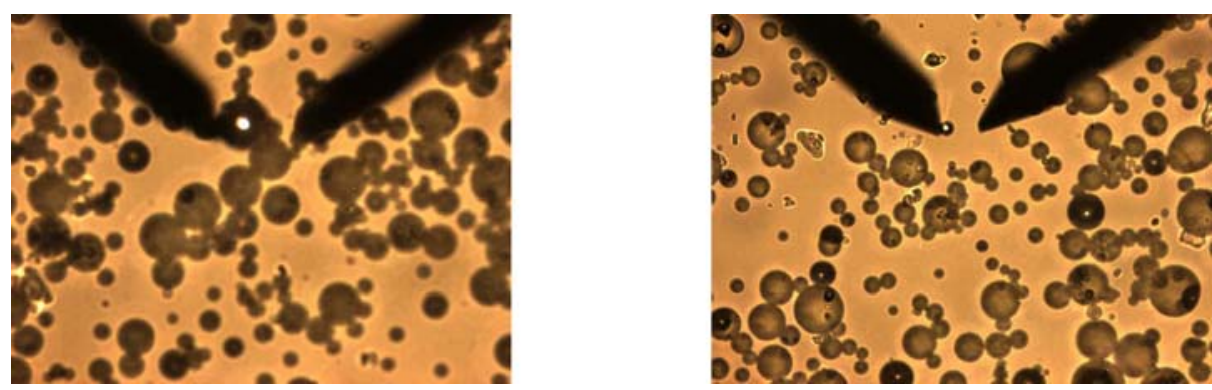

Fig. 5. Glass sphere adhesion during micromanipulation task. Rigth: small sphere near $3 \mu \mathrm{m} \$$ and Left: big sphere near $45 \mu \mathrm{m}$.

The adhesion can be cancelled by functionalisation of the grippers and the spheres. 


\subsection{Functionalised Objetcs}

In a previous paper, we have shown a repulsion between the surface and cantilever when both are functionalised by an aminosilane and this whatever the $\mathrm{pH}$ of the solution. In this paper, illustration of this phenomena is shown in micromanipulation tasks. As the repulsive force has a high interaction distance (typicallytens of micrometers) [9], this effect can be used to manipulate the object without contact with the end-effector. Indeed, when the functionalized end-effector is approached near to the functionalized object, the object is moving behind the end-effector (Figure 6). When the ball is caught, the sphere release is easy because of the repulsive force. This release is illustrated Figure 7. In this Figure, we observe that the adhesion does not disturb the release.

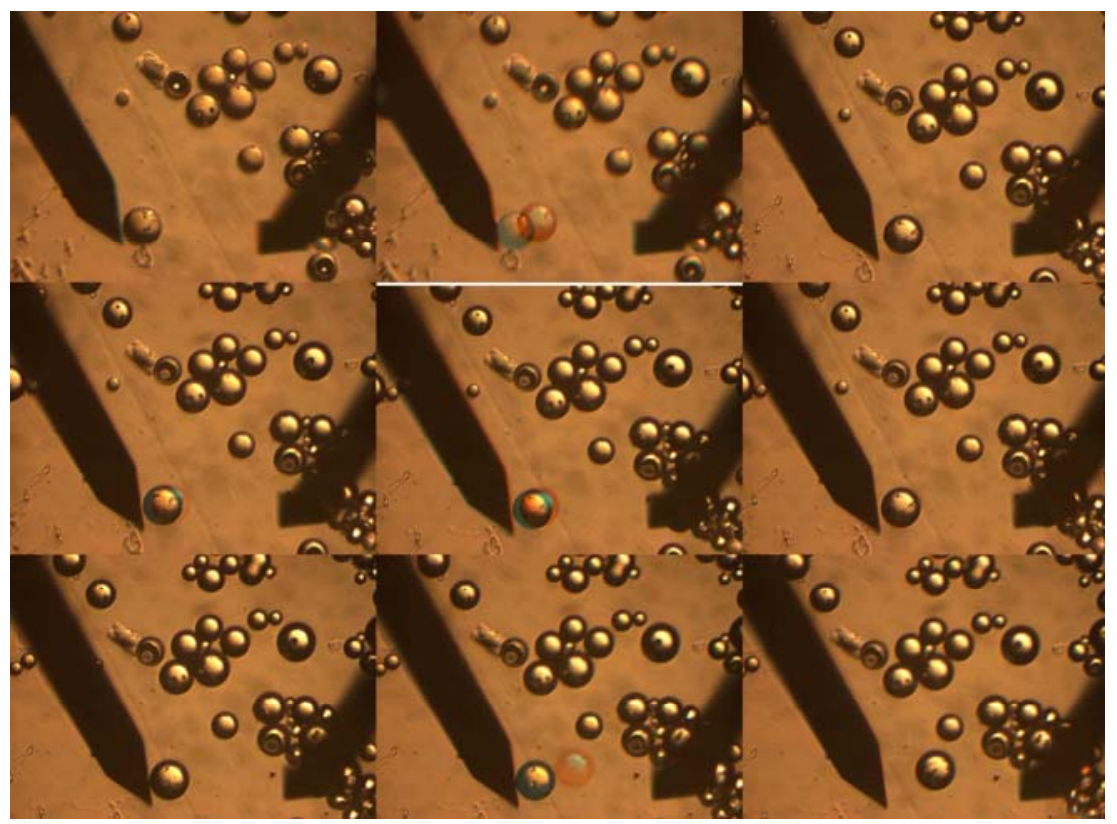

Fig. 6. Repulsion of the functionalised glass sphere when a functionalised SiFit approach to its

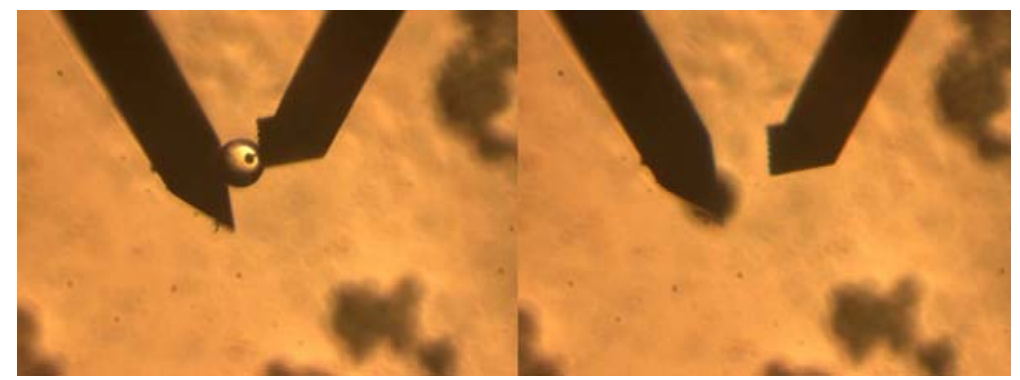

Fig. 7. Release of functionalised glass sphere when the functionalised gripper open 


\section{Conclusion}

In this paper we have observed the adhesion behaviour between two functionalised surfaces and between two surfaces. We have shown the correlation between the forcedistance measurement and the micromanipulation tacks. The liquid medium and the chemical functionalisation can be used to control the surface properties and the microobject release. As adhesion is the current highest disturbance in micromanipulation, functionalisation is a promising way to improve micro-object manipulation in the future.

\section{Acknowledgement}

This work was supported by the EU under HYDROMEL contract NMP2-CT-2006026622 : Hybrid ultra precision manufacturing process based on positional- and selfassembly for complex micro-products, and by the French National Agency (ANR) under NANOROL contract ANR-07-ROBO-0003: Nanoanalyse for micromanipulate.

\section{References}

[1] Lambert, P.: Capillary Forces in Micro-assembly. Springer, Heidelberg (2008)

[2] Gauthier, M., Régnier, S., Rougeot, P., Chaillet, N.: Forces analysis for micromanipulations in dry and liquid media. Journal of Micromechatronics 3, 389-413 (2006)

[3] Dove, P., Craven, C.: Surface charge density on silica in alkali and alkaline earth chloride electrolyte solutions. Geochimica et Cosmochimica Acta 69, 4963-4970 (2005)

[4] Dejeu, J., Lakard, B., Fievet, P., Lakard, S.: Characterization of charge properties of an ultrafiltration membrane modified by surface grafting of poly(allylamine) hydrochloride. Journal of Colloid and Interface Science 333, 335-340 (2009)

[5] Wang, J., Guo, D., Xia, B., Chao, J., Xiao, S.: Preparation of organic monolayers with azide on porous silicon via sin bonds. Colloids and Surface A: Physicochemical and Engineering Aspects 305, 66-75 (2007)

[6] Wasserman, S., Tao, Y., Whitesides, G.: Structure and reactivity of alkylsiloxane monolayers formed by reaction of alkyltrichlorosilanes on silicon substrates. Langmuir 5, 1074-1087 (1989)

[7] Ulman, A.: An Introduction to Ultrathin Organic Films From Langmuir-Blogett to SelfAssembly. Academic Press, London (1991)

[8] Dejeu, J., Rougeot, P., Gauthier, M., Boireau, W.: Adhesions forces controlled by chemical self-assembly and ph, application to robotic microhandling. ACS Applied Materials \& Interfaces 1, 1966-1973 (2009)

[9] Dejeu, J., Rougeot, P., Gauthier, M., Boireau, W.: Robotic submerged microhangling controlled by ph switching. In: Proc. of the IEEE IROS 2009 conference, St Louis (2009)

[10] Vinogradova, O., Yakubov, G.: Dynamic effects on force measurements. 2. lubrication and the atomic force microscope. Langmuir 19, 1227-1234 (2003)

[11] Agnus, J., Hériban, D., Gauthier, M., Pétrini, V.: Silicon end-effectors for microgripping tasks. Precision Engineering 33, 542-548 (2009) 